\title{
A Fast Low-Noise Charged-Particle CVD Diamond Detector
}

\author{
H. Frais-Kölbl, E. Griesmayer, Member, IEEE, H. Kagan, and H. Pernegger
}

\begin{abstract}
This paper presents the design and test results of a charged-particle solid-state detector with ultrafast signal response based on polycrystalline chemical-vapor-deposition (pCVD) diamond as active detector material and a high-bandwidth RF amplifier. We tested the detector at the Indiana University Cyclotron Facility Bloomington, IN, in a proton beam with a kinetic energy ranging from 55 to $200 \mathrm{MeV}$. The detector signals showed an average pulsewidth of $1.38 \mathrm{~ns}$, which enables single-particle counting at instantaneous rates approaching the gigahertz range. The detector operated with a signal-to-noise ratio of $7: 1$ for $200-\mathrm{MeV}$ protons and a single-particle detection efficiency up to $99 \%$.
\end{abstract}

Index Terms-Biomedical nuclear imaging, diamond, high-speed electronics, proton accelerators, proton detectors, semiconductor radiation detectors.

\section{INTRODUCTION}

W E developed and tested a solid-state detector for very high particle rates. As part of the European Organization for Nuclear Research (CERN), Geneva, Switzerland, RD42 test program, we built the detector system for possible use in the beam diagnostic system of MedAustron, a proposed medical accelerator facility for ion-radiation cancer therapy [2]. The detector system is based on polycrystalline chemical-vapor-deposition (pCVD) diamond [1] as active sensor material and analog front-end electronics operating in the gigahertz range. The compact solid-state detector produces fast signals which enables the detection of single particles at rates approaching the gigahertz range per channel. Detectors with these properties have applications in medical instrumentation, instrumentation in the fields of particle and nuclear physics, or as a beam condition monitor.

Advantages of diamond detector material are that no doping is required as in silicon detectors, that it can be operated at leakage currents of less than $1 \mathrm{nA}$ because of its high intrinsic resistivity, that the ionization current pulses are very fast due to the high charge-carrier velocity combined with the short life time and that the material is radiation hard at fluences exceeding $2 \cdot 10^{15}$ protons $/ \mathrm{cm}^{2}$ [3].

The fast signal response of natural and standard quality CVD diamonds with charge collection distances of tens of micrometers is well established [4]-[6]. These diamonds have previously been combined with broadband amplifiers for example

Manuscript received February 23, 2004; revised June 28, 2004.

H. Frais-Kölbl and E. Griesmayer are with the Fachhochschule für Wirtschaft und Technik, A-2700 Wiener Neustadt, Austria.

H. Pernegger is with the Department of Physics, European Organization for Nuclear Research, CH-1211 Geneva, Switzerland.

H. Kagan is with Department of Physics, The Ohio State University, Columbus, OH 43210 USA.

Digital Object Identifier 10.1109/TNS.2004.839366 for heavy-ion detection [7], [8]. These applications or measurements are typically characterized by large energy deposition in the diamond, therefore, even moderate noise performance of the readout electronics is sufficient for reliable particle detection.

The focus of our work is to use high-quality CVD diamonds, with a charge collection distance well above $100 \mu \mathrm{m}$, as fast particle detectors for applications with low, few times minimum ionizing energy deposition and develop suitable high-bandwidth electronics with high amplification and very low noise. Further work is currently ongoing to extend the method to the detection of single minimum-ionizing particles with signals in the nanosecond range, which will make it ideally suited for a large range of beam diagnostic systems.

In particle-detection applications pCVD diamonds are usually plated with electrodes ranging from a few micrometers to several centimeters in size with bulk thicknesses of $500 \mu \mathrm{m}$ or less. The flexibility in choice of electrode geometry allows position sensitive structures on a single detector as well as dose measurements. An overview of properties of CVD diamond detectors can be found in [9] and [10].

\section{CVD DIAMOND DETECTOR}

The CVD diamond used for this test has been developed by the CERN RD42 collaboration in cooperation with Element Six Ltd., Ascot, U.K. The detector diamond measures $10 \times 10 \mathrm{~mm}^{2}$ and is $500 \mu \mathrm{m}$ thick. Its surfaces were mechanically polished prior to metallization. Subsequently the substrate is plated with square $\mathrm{Cr} / \mathrm{Au}$ electrodes on the top and bottom of $7.5 \times 7.5 \mathrm{~mm}^{2}$ by evaporation and annealed at $400{ }^{\circ} \mathrm{C}$ for $5 \mathrm{~min}$. The chargecollection distance is $\delta=190 \mu \mathrm{m}$, equivalent to a mean charge signal of 6800 electron-hole pairs for a minimum-ionizing particle, which generate a charge of $\mathrm{q}_{M}=36 \mathrm{e}-\mathrm{h}$ pairs per micrometer of path length [11]. We measured the diamond charge collection distance using a collimated ${ }^{90} \mathrm{Sr}$ source and readout the CVD diamond through a calibrated charge-sensing amplifier. Further details of method and setup are described in [11]. A grounded guard ring on the top side precisely defines the sensitive area. The top side of the pad, which is placed on the diamond substrate side, is connected to the ground through a 1-M $\Omega$ resistor and ac coupled to the preamplifier through a 1-nF coupling capacitance. The bottom side of the pad is connected to the bias voltage. The diamond detector was operated at a bias voltage of $950 \mathrm{~V}$ equivalent to an electric field of $1.9 \mathrm{~V} / \mu \mathrm{m}$.

\section{DETECTOR EleCtronics}

We designed a preamplifier without feedback in order to amplify the detector-current pulse. This design allows a higher 


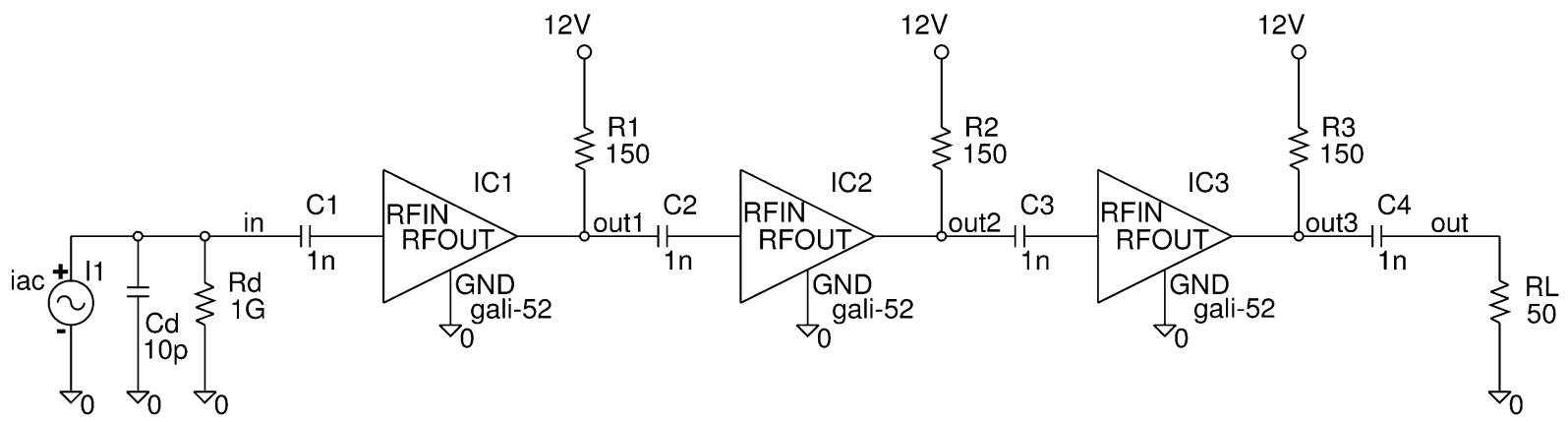

Fig. 1. Schematics of the preamplifier consisting of three stages. $R_{d}$ and $C_{d}$ denote equivalent resistance and capacitance of the detector. Details of the detector connection are not shown in the schematics but described in the text.

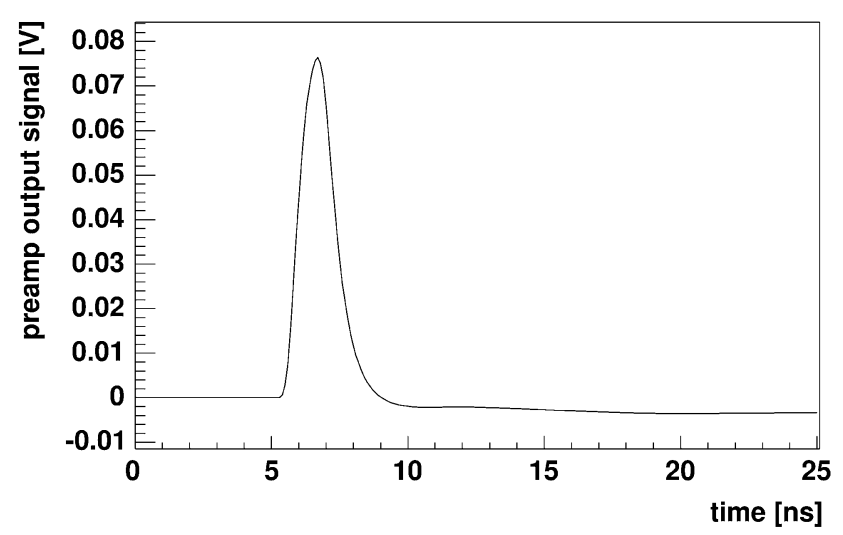

Fig. 2. Simulated pulse response of an MIP signal with an FWHM of $1.4 \mathrm{~ns}$.

bandwidth compared to a charge-sensitive amplifier and makes the electronics noise independent of the detector capacitance. We used an RF amplifier with a bandwidth of $2 \mathrm{GHz}$. The preamplifier is comprised of three stages with 20 -dB gain each, resulting in a total gain of $60 \mathrm{~dB}$.

A schematic of the preamplifier is shown in Fig. 1. The three stages are ac-coupled with $1-n F$ coupling capacitors, resulting in an overall lower cutoff frequency of approximately $3 \mathrm{MHz}$. The dc is applied via $150-\Omega$ resistors. A simulated pulse response obtained in a SPICE simulation of the RF amplifier is shown in Fig. 2. For the RF amplifier we used high-gain and low-noise Gali-52 chips from MiniCircuit.

The specifications of the RF amplifiers are:

- signal bandwidth $B=2 \mathrm{GHz}$;

- signal amplification $G=20 \mathrm{~dB}$;

- noise figure $F=2.7 \mathrm{~dB}$.

The parallel noise figure for three amplifier stages is

$$
F_{\text {tot }}=F+\frac{F-1}{G}+\frac{F-1}{G^{2}}=1.87
$$

which results in the equivalent noise power at the input of the amplifier

$$
N_{i}=\left(F_{\text {tot }}-1\right) n_{0} B \frac{3 \pi}{16}=4.1 \mathrm{pW}
$$

where $n_{0}=4 \cdot 10^{-21} \mathrm{~W} / \mathrm{Hz}$ denotes the spectral power density of white thermic noise. The input noise can be expressed as rms noise current

$$
i_{N_{i}}=\sqrt{\frac{N_{i}}{R_{i}}}=286 \mathrm{nA}
$$

\section{SignAL ESTIMATION}

In the following we provide an estimation of the initial-current signal of a minimum-ionizing particle (MIP). For an approximate evaluation of the expected signal-to-noise ratio, we use the estimated signal and the parallel noise of above. It should be noted that the serial noise is omitted in the noise calculation.

The ionization charge drifts in the electrical field and recombines with charge traps leading to an effective charge-collection distance $\delta$. For the estimation, given our large operating electric field, we use the saturated electron and holes drift velocity of $v_{e}=180 \mu \mathrm{m} / \mathrm{ns}$ and $v_{h}=120 \mu \mathrm{m} / \mathrm{ns}$, respectively [9].

The drift induces a current pulse at the electrodes. The induced initial current $i_{0}$ can be calculated by the Shockley-Ramo Theorem [12], [13] for a uniform constant field between the two electrodes as

$$
i_{0}=q \cdot \frac{v}{d}
$$

where $q$ denotes the total ionization charge, $v$ the drift velocity, and $d$ the gap between the electrodes, which is equal to the thickness of the detector. The total initial ionization charge is $q=q_{M} \cdot d$ which yields an incident detector current of

$$
i_{0}=q_{M} \cdot v_{e}+q_{M} \cdot v_{h}=1.73 \mu \mathrm{A}
$$

which is independent of the detector thickness $d$ and the chargecollection distance $\delta$. The input resistance $R_{i}$ of the RF amplifier is $50 \Omega$ and the input voltage for an MIP signal is $u_{i}=86.4 \mu \mathrm{V}$ which is amplified by $60 \mathrm{~dB}$ to $u_{0}=86.4 \mathrm{mV}$.

The pulsewidth $\tau$ can be estimated from the charge-collection distance and the mean drift velocity by

$$
\tau=\frac{2 \delta}{v_{e}+v_{h}}=1.27 \mathrm{~ns} .
$$

The ratio of the peak signal to the rms noise, which is denoted as signal-to-noise ratio (SNR) can be calculated for an MIP as

$$
\mathrm{SNR}=\frac{i_{0}}{i_{N_{i}}}=6.07
$$

which corresponds to a theoretical mean SNR of 36 for $55 \mathrm{MeV}$, and 14 for $200 \mathrm{MeV}$ protons, respectively.

\section{Results OF THE BEAM TEST}

The detector was tested at the Radiation Test Facility of the Indiana University Cyclotron Facility (IUCF), Bloomington, IN, which provided a proton beam with a kinetic energy 


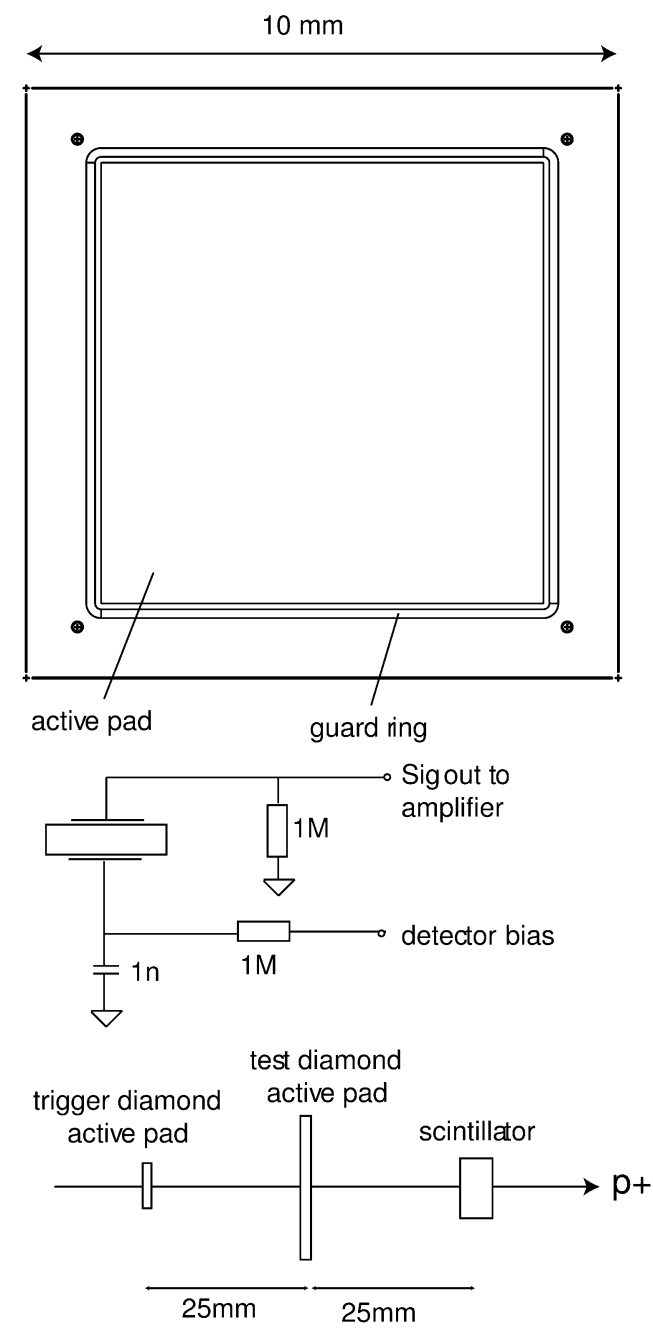

Fig. 3. Layout of diamond detector pad and guard ring (top), the detector bias circuit used during the test (middle) and schematic illustration of detector arrangement during the beam test (bottom, not to scale).

ranging from 55 to $200 \mathrm{MeV}$ and adjustable intensities which match medical treatment conditions. The detector diamond was mounted and carefully aligned upstream of a trigger scintillator with an active area of $5 \times 5 \mathrm{~mm}^{2}$ and a trigger diamond detector with a pad size of $2.5 \times 2.5 \mathrm{~mm}^{2}$. Fig. 3 shows the layout of the detector diamond together with its alignment in the testbeam and detector bias circuit.

The trigger diamond uses an identical setup and read-out electronics as the diamond under test. The preamplifier was located close to the diamond detector. The output of the preamplifier for the diamond under test and the trigger diamond was connected by a 15-m-long shielded cable to a LeCroy WaveMaster 8600 digital oscilloscope with an analog bandwidth of $6 \mathrm{GHz}$ and a sampling frequency of 20 GSPS. For timing measurements the scope was located close to the preamplifier and connected by a 1-m cable.

The detector was exposed to beam energies of 200, 150, 105, 75 , and $55 \mathrm{MeV}$, which covers the typical proton energy range for medical accelerators. This energy range corresponds to a theoretical mean energy loss in diamond of 2.3-5.9 times minimum ionizing. The typical flux during the measurements was $10^{8}$ protons $/ \mathrm{cm}^{2} \mathrm{~s}$.
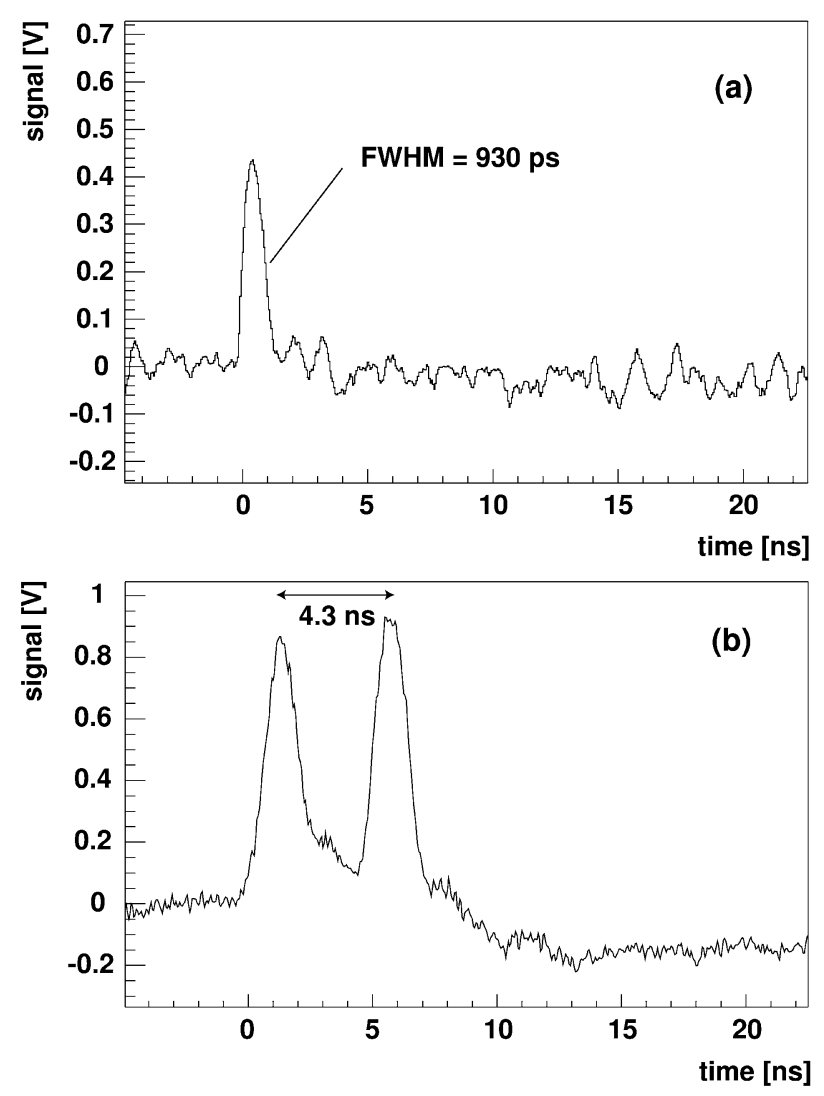

Fig. 4. Signals at the output of the preamplifier. (a) Single pulse with an FWHM of 0, 93 ns. (b) Double pulse with a double-pulse separation of $4.3 \mathrm{~ns}$.

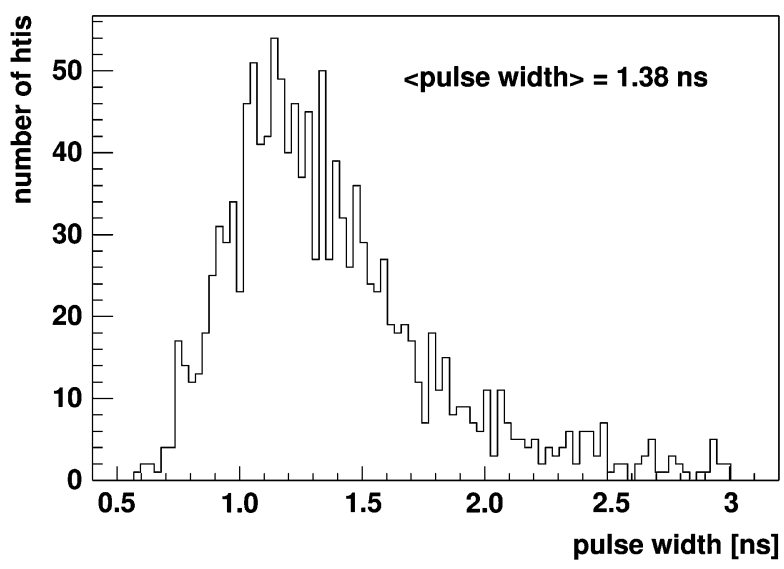

Fig. 5. Measured FWHM-pulsewidth distribution.

\section{A. Signal Response and Timing Characteristics}

For measuring the properties of the detector, we recorded 50-ns-long sequences of detector signals which were triggered by the coincidence of the scintillator signal and the trigger diamond. Fig. 4 shows the signal response of a single pulse [Fig. 4(a)] and a double pulse [Fig. 4(b)], where two particles traverse the diamond detector within $4.3 \mathrm{~ns}$. The full-width at half-maximum (FWHM) pulsewidth distribution is shown in Fig. 5. This distribution starts at 600 ps and shows an average value of $1.38 \mathrm{~ns}$. The average rise time is $350 \mathrm{ps}$. Shorter pulses are weakly correlated with pulses of lower amplitude. 

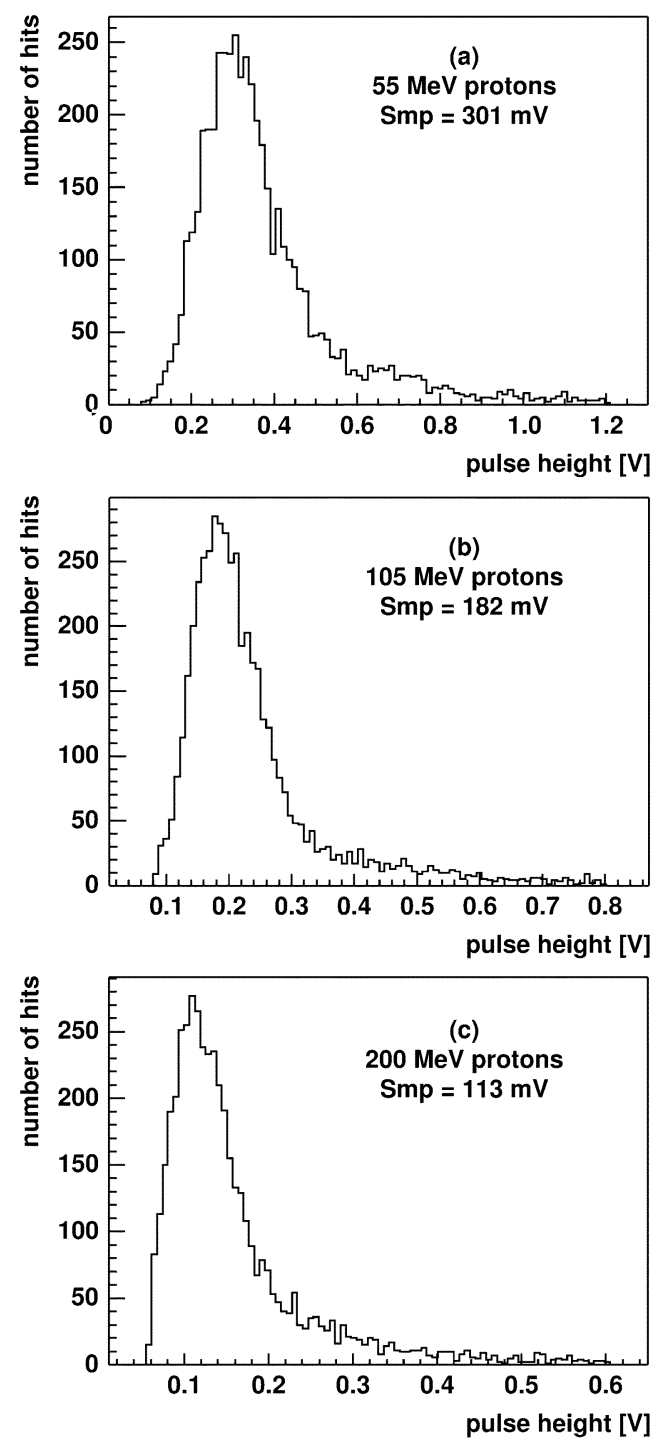

Fig. 6. Measured pulse-height distributions for 55-, 105-, and 200-MeV protons and the corresponding Landau fits (dashed lines).

\section{B. Amplitude Distribution and Energy Dependence}

Fig. 6 shows measured amplitude distributions for three different beam energies. The distributions follow the expected Landau characteristics. At the measurement energies of 55, 75, 105,150 , and $200 \mathrm{MeV}$ we find most probable amplitudes of $301,228,182,142$, and $113 \mathrm{mV}$, respectively. For the same energies we recorded a most probable SNR of 14.5: 1, 11.6:1, $9.2: 1,7.2: 1$, and $7.1: 1$, respectively.

Fig. 7 shows the most probable signal amplitude as a function of the kinetic beam energy and compares it to the calculated Bethe-Bloch energy loss [14]. Due to the uncertainty of the calibration for our diamond detector we normalized the calculation to the test data at $105 \mathrm{MeV}$. Vertical error bars show the error on the most probable signal extracted from a convoluted Landau-Gauss fit to the data, horizontal error bars indicate the uncertainty in beam energy from the accelerator. The plot clearly shows the decrease of signal amplitude with increasing beam energy, which is consistent with the expected behavior.

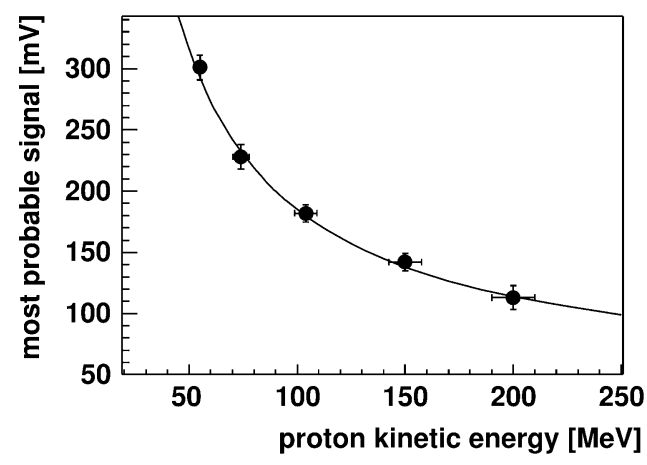

Fig. 7. Measured and calculated dependence of the most probable signal amplitude as a function of the proton kinetic energy.

The measured values for signal amplitudes and the width of the signals correspond well to the estimation in Section IV.

\section{Detector Efficiency}

The counting efficiency of the detector was determined as the ratio of number of events with signals on the test diamond to the number of trigger events. We rejected signals with amplitudes less than three times baseline noise. The detector shows an efficiency of $99.9 \%$ at $55 \mathrm{MeV}, 99.6 \%$ at $105 \mathrm{MeV}$, and $96.8 \%$ for $200 \mathrm{MeV}$, respectively.

It should be noted that the detection efficiency is limited by the electronic noise rather than the detector material, as the diamond is close to $100 \%$ efficient at this charge collection distance and thickness. The change of efficiency is a direct consequence of the decreasing signal amplitude with increasing beam energy at constant noise level.

\section{CONCLUSION}

Combining the fast signal response of a high-quality CVD diamond detector with a fast low-noise RF amplifier allowed us to develop a charged-particle detector with very fast signal response and low noise level. Further work is ongoing to increase the sensitivity to the detection of single minimum-ionizing particles while maintaining the speed characteristics. Analysis of the pulse shape suggests that the detector is capable of counting single charged particles at rates approaching the gigahertz range. We measured an average rise time of 350 ps and a mean pulsewidth of $1.38 \mathrm{~ns}$. The amplitude distribution shows the expected Landau characteristics and energy dependence in the energy range of 55-200 $\mathrm{MeV}$ protons. The most probable SNR was 7: 1 for $200 \mathrm{MeV}$ protons. The efficiency was $96.8 \%$ at $200 \mathrm{MeV}$ and $99.9 \%$ at $55 \mathrm{MeV}$, respectively. This clearly demonstrates the functionality of the detector for applications as ultrafast particle counter for protons in this energy range.

\section{ACKNOWLEDGMENT}

The authors want to thank the IUCF Radiation Effects Research Program staff including Dr. S. B. Klein, as well as Dr. C. C. Foster of Foster Consulting Services, L.L.C., and K. M. Murray of KM Sciences, for their time and assistance in conducting the proton irradiation described in this paper. The proton beam delivery time was granted by the IUCF in consideration 
of the potential for sharing mutually beneficial detector technology. This work was supported by the county of Lower Austria and the town of Wiener Neustadt, Austria, in the frame of the project MedAustron.

\section{REFERENCES}

[1] "Development of diamond tracking detectors for high luminosity experiments at LHC," The RD42 Collaboration, Geneva, Switzerland, R\&D Proposal, DRDC/P56, CERN/DRDC 94-21, 1994.

[2] R. Pötter et al., "MedAustron Machbarkeitsstudie," Abteilung Kultur und Wissenschaft des Amtes der NÖ Landesregierung und dem Regional-Innovationszentrum, Niederösterreich Süd, Austria, 1998.

[3] W. Adam et al., "Development of CVD diamond radiation detectors," Proc. Electrochem. Soc., vol. 97-32, pp. 491-497, 1997.

[4] S. Han et al., "Temporally resolved response of natural IIa diamond detector to single particle exitation," Diamond Related Mater, vol. 2, pp. 835-840, 1993.

[5] M. Nail et al., "Experimental results in picoseconds and subnanoseconds range of IIa type diamond detectors in X-UV, visible and IR fields," in Proc. Materials Research Soc. Symp. V, vol. 302, 1993, pp. 263-273.
[6] S. Han, R. S. Wagner, J. Joseph, M. A. Plano, and M. D. Moyer, "Chemical vapor deposited diamond radiation detectors for ultrahigh radiation dose-rate measurements: Response to subnanosecond, $16-\mathrm{MeV}$ electron pulses," Rev. Sci. Instrum., vol. 66, pp. 5516-5521, 1995.

[7] P. Moritz, E. Berdermann, K. Blasche, H. Stelzer, and B. Voss, "Broadband electronics for CVD diamond detectors," Diamond Related Mater. vol. 10, pp. 1765-1769, 2001

[8] E. Berdermann, K. Blasche, P. Moritz, H. Stelzer, and B. Voss, "The use of CVD-diamond for heavy-ion detection," Diamond Related Mater. vol. 10, pp. 1770-1777, 2001.

[9] M. Franklin et al., "Development of diamond radiation detectors for SSC and LHC," Nucl. Instrum. Methods, vol. A315, pp. 39-42, 1992.

[10] P. Bergonzo, D. Tromson, C. Mer, B. Guizard, F. Foulon, and A. Brambilla, "Particle radiation detectors based on diamond," Phys. Stat. Sol. (a), vol. 185, pp. 167-181, 2001.

[11] S. Zhao, "Characterization of the electrical properties of polycrystalline diamond films," Ph.D. dissertation, The Ohio State University, Columbus, OH, 1994.

[12] W. Shockley, "Currents to Conductors Induced by a Moving Point Charge," J. Appl. Phys., vol. 9, pp. 635-636, Oct. 1938.

[13] S. Ramo, "Currents Induced by Electron Motion," in Proc. I.R.E., vol. 27, Sept. 1939 , pp. 584-585.

[14] M. J. Berger, J. S. Coursey, and M. A. Zucker. (2004, Jan. 17) ESTAR, PSTAR, and ASTAR: Computer Programs for Calculating Stopping-Power and Range Tables for Electrons, Protons, and Helium Ions (version 1.2.2) [Online]. Available: http://physics.nist.gov/Star 